International Journal of Modern Physics B

Vol. 32, No. 7 (2018) 1850075 (22 pages)

(C) The Author(s)

DOI: $10.1142 / \mathrm{S} 0217979218500753$

\title{
Dynamics of gene expression with positive feedback to histone modifications at bivalent domains
}

\author{
Rongsheng Huang and Jinzhi Lei* \\ Zhou Pei-Yuan Center for Applied Mathematics, \\ MOE Key Laboratory of Bioinformatics, \\ Tsinghua University, Beijing 100084, P. R. China \\ *jzlei@tsinghua.edu.cn
}

Received 21 June 2017

Revised 6 October 2017

Accepted 31 October 2017

Published 16 November 2017

\begin{abstract}
Experiments have shown that in embryonic stem cells, the promoters of many lineagecontrol genes contain "bivalent domains", within which the nucleosomes possess both active (H3K4me3) and repressive (H3K27me3) marks. Such bivalent modifications play important roles in maintaining pluripotency in embryonic stem cells. Here, to investigate gene expression dynamics when there are regulations in bivalent histone modifications and random partition in cell divisions, we study how positive feedback to histone methylation/demethylation controls the transition dynamics of the histone modification patterns along with cell cycles. We constructed a computational model that includes dynamics of histone marks, three-stage chromatin state transitions, transcription and translation, feedbacks from protein product to enzymes to regulate the addition and removal of histone marks, and the inheritance of nucleosome state between cell cycles. The model reveals how dynamics of both nucleosome state transition and gene expression are dependent on the enzyme activities and feedback regulations. Results show that the combination of stochastic histone modification at each cell division and the deterministic feedback regulation work together to adjust the dynamics of chromatin state transition in stem cell regenerations.
\end{abstract}

Keywords: Epigenetic regulation; stochastic modeling; gene expression; inheritance; cell differentiation.

PACS numbers: 87.16.Sr, 87.17.Aa, 87.18.Tt

This is an Open Access article published by World Scientific Publishing Company. It is distributed under the terms of the Creative Commons Attribution 4.0 (CC-BY) License. Further distribution of this work is permitted, provided the original work is properly cited.

* Corresponding author. 


\section{Introduction}

In eukaryotic cells, most DNA sequences are enclosed into basic organizational chromatin units-nucleosomes. A typical nucleosome has approximately 147 nucleotide base pairs that wrap around a histone octamer; the octamer is composed of one $(\mathrm{H} 3-\mathrm{H} 4)_{2}$ tetramer capped by two H2A-H2B dimers. ${ }^{6}$ Histone N-termini (tails) can undergo diverse post-translational covalent modifications, including acetylation, phosphorylation, methylation, ubiquitination and ADP-ribosylation ${ }^{13}$; these modifications can lead to either active or repressive gene expression activities. ${ }^{2,11}$ A dynamic equilibrium of histone modifications can be attained due to the balance between various enzymes that are associated with the addition and removal of covalent marks.

Experiments suggest that the nucleosome covalent patterns can be transmitted over a number of generations. Molecular details of nucleosome modifications during cell division have been extensively studied. ${ }^{20}$ During DNA replication, parental histones $\mathrm{H} 3$ and $\mathrm{H} 4$ that carry post-translational modifications can be distributed onto daughter strands in three possible ways, random distribution, semi-conservative distribution, or asymmetric distribution; following which the nucleosome modifications are rebuilt with the incorporation of newly synthesized histones. The parental marks are recognized by corresponding chromatin-binding proteins, reader proteins that recruit chromatin modifiers, or writer proteins that restore the marks at daughter strands. ${ }^{22}$ This read-and-write process is less reliable than the maintenance of DNA sequence, so there are fluctuations in the nucleosome modification patterns between daughter cells and the mother cell at each cell division. The dynamical changes of nucleosome modification patterns are associated with embryo development and stem cell differentiation. ${ }^{4,29}$ In embryo development, there are bivalent domains that consist of both repressive marks and active marks. 1 These bivalent domains tend to coincide with genes expressed at low-level, however keeping them poised for activation, and switch to either active or repressive marks dominant in further development.

Many computational models have been proposed to study the dynamics of nucleosome modification patterns. Dodd et al. $\frac{5}{5}$ shows that cooperative effects beyond neighboring nucleosomes can lead to the robust bistability due to compact chromatin structures. Moreover, a transcription factor (TF) binding noncooperatively to a single site can change the balance between opposing positive feedback loops in histone modifications to produce ultrasensitive response in gene expression. $^{25}$ A statistical mechanics model of collective epigenetic histone modification suggested that nearest-neighbor enzyme lateral interactions and nucleosome modification state-biased enzyme recruitment together can lead to effective nonlocal interactions among nucleosomes and inheritable epigenetic memory. ${ }^{28}$ The dynamics of histone modifications with cooperative interactions between multiple marks and the modifying enzymes are discussed with computational models with different construction of histone state transitions. ${ }^{12,19,24}$ Simulations reveal that inheritable 
chromatin states can be formed following cooperative interactions between histone covalent modifications and modifier enzymes. Despite extensive studies about the dynamics of histone modifications, quantitative modeling of how nucleosome state controls gene expression in single cells is considered less. Experimentally, time-lapse microscopy analyzing at single-cell level has shown that silencing and reactivation of repressive marks can modulate the fraction of cell silence rather than the amount of gene expression. ${ }^{2}$

In this study, to investigate how nucleosome state transition in bivalent domains can be regulated with enzyme activities, we considered the feedback regulation between gene product and enzyme activities; gene products regulate histone covalent modification of nucleosomes state within its own promoter region. We constructed a hybrid computational model that includes dynamics of histone marks, three-stage chromatin state transitions, gene expression, feedbacks from protein product to enzymes regulating the addition and removal of histone marks, and inheritance of nucleosome state in cell division. The model reveals how both nucleosome state transition and gene expression are dependent on the enzyme activities and feedback regulations.

\section{Materials and Methods}

\subsection{Dynamics of histone modification}

We referred $\mathrm{Ku}$ et al. $\frac{12}{}$ for the model of the dynamics of histone modification. Here, we attempted to study the effects of histone modifications on gene expression dynamics in eukaryotic system. Despite usual diploid karyotype in eukaryotes, here we mainly considered a model based on haploid karyotype. The fact that diploid karyotype may introduce an additional complexity to the mRNA concentration dynamics however would not change the effects of histone dynamics. The model describes the promoter activities of a gene that contains $N$ nucleosomes in the promoter region. Each nucleosome contains two copies of the core histone H3, whose tail can undergo various types of covalent modifications, such as methylation and acetylation. Two types of methylations, the trimethylation of H3 lysine 4 (H3K4me3) and the trimethylation of H3 lysine 27 (H3K27me3), are of particular interest in this study. H3K4me3 is often associated with active transcription, and H3K27me3 is associated with transcription repression. Each H3 histone can be in one of the states of unmodified (U), modified by an active mark H3K4me3 (A), or modified by a repressive mark H3K27me3 (R). ${ }^{12}$ Hence, each nucleosome can be in one of the six physically distinct nucleosome states, UU, AU, UR, AA, AR, RR [Fig. 1(A)]:

- UU: The two H3 histones are unmodified.

- AU: One H3 histone is modified with an active mark, and the other one is unmodified.

- UR: One H3 histone is modified with a repressive mark, and the other one is unmodified. 
(A)

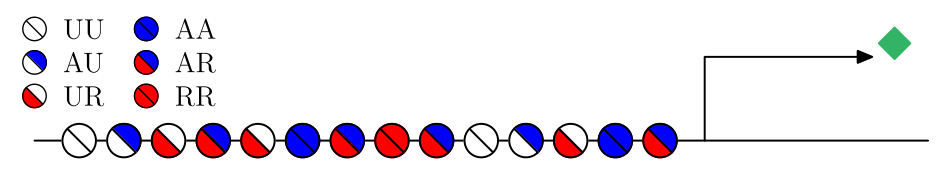

Promoter

(B)

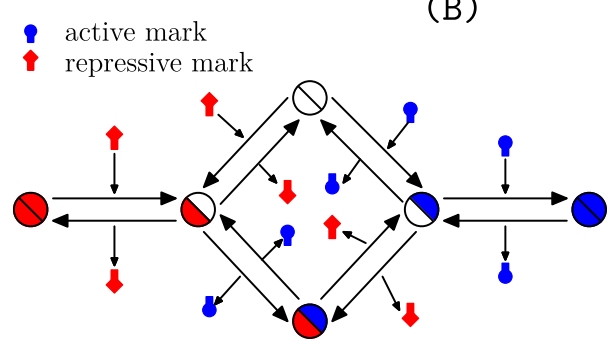

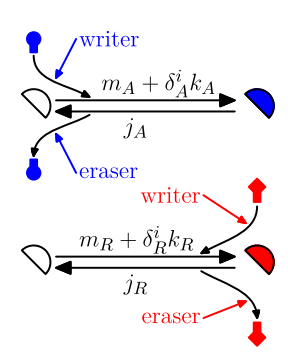

Fig. 1. (Color online) Illustration of the gene transcription regulated by histone modification. (A) The promoter region contains many nucleosomes, each nucleosome can be in one of the six possible states: UU, AU, UR, AA, AR, RR. The transcription rate is dependent on the overall nucleosome states (refer Fig. 2). (B) Dynamics of nucleosome state transition. The six nucleosome states transit between each other through the chemical reactions of methylation/demethylation, as shown to the left panel. The right panel shows the reactions of adding/removing an active mark (upper panel) or repressive mark (bottom panel), which are regulated by the corresponding enzymes. Refer to the text for details.

- AA: Both H3 histones are modified with active marks.

- AR: One H3 histone is modified with an active mark, and the other one is unmodified.

- RR: Both H3 histones are modified with repressive marks.

Here, the state AR means bivalency.

The nucleosome states are dynamically changing due to chemical reactions of methylation/demethylation, which are regulated by the corresponding enzymes [Fig. 1(B)]. The involved biochemical reactions can be classified into four types of reactions: the methylation of unmodified sites $(\mathrm{U})$ with the active $(\mathrm{A})$ or repressive $(\mathrm{R})$ markers, or the demethylation of modified sites (active or repressive) [Fig. 1(B), right panel]. In the model, we introduced following assumptions for the formulation of reaction rates:

- Upon methylation, the modified site is recognized by a chromatin-binding protein, a reader protein, which in turn recruits other proteins that lead to the additional chromatin-modifying activities (including "writer" and "eraser" proteins). The writer induces the methylation of the neighboring nucleosome, and the eraser removes the methyl group from the methylated nucleosome ${ }^{20}$. Thus, both demethylation rates $j_{X}(X=A, R)$ and methylation rates $k_{X}(X=A, R)$ are dependent on the corresponding enzyme activities. Moreover, the methylation 
rates are mediated by the neighboring nucleosome that $k_{X}$ also depends on the state of the neighboring nucleosome.

- To specify the neighboring state-mediated methylation, for each nucleosome, we introduced a pre-factor $\delta_{X}^{i}(X=A, R)$ for the state of its neighboring nucleosome, which is defined as

$$
\delta_{X}^{i}= \begin{cases}1, & \text { if the }(i-1) \text { or }(i+1) \text { nucleosome } \\ & \text { contains a marker } X \\ 0, & \text { otherwise. }\end{cases}
$$

- Here, a nucleosome contains a mark A or R if one H3 histone has the mark.

- In addition to the neighboring state-mediated methylation, each unmodified nucleosome can be methylated with a basal methylation rate $m_{X}(X=A, R)$. Thus, the effective methylation rate is given by $m_{X}+\delta_{X}^{i} k_{X}$.

- During cell division, when DNA replication occurs, each copy of the H3 histone in the parental nucleosomes is randomly assigned to one of the two daughter strands. Thus, in a daughter strand, when a nucleosome inherits histones from the parental strand, it also inherits the histone modification marks. The newly synthesized histones are assigned with unmodified states. ${ }^{20}$

These assumptions together lead to a numerical scheme of stochastic simulation for the kinetics of histone modifications over multiple cell cycles.

\subsection{Chromatin state and active transcription}

Histone modifications play important roles in the modulation of gene expression. However, the molecular mechanisms of how they control gene expression remain unclear. A three-state model to explain gene expression dynamics under epigenetic regulation was recently established based on cell-to-cell variability at the singlecell level. ${ }^{2}$ In this three-state model, the chromatin stochastically transits between three states: active expression (A), reversibly silent (R), and irreversibly silent (I); the transition kinetics between chromatin states are mediated by the nucleosome states. This three-state model is the basis to connect nucleosome modification with gene expression.

In the three-state model, the irreversible silent state is an attractor so that the gene is eventually silent, which might be too strong for some genes unless the silent state can be maintained by some other mechanisms. Usually, H3K27me3 is associated with the repression of gene transcription, and a gene can further be silenced by DNA methylation. In this study, DNA methylation was not included due to the yet unclear collaborative dynamics between DNA methylation and histone modifications. $\frac{26}{}$ Alternatively, we introduced an assumption that repressive modifications (UR or RR) are able to induce chromatin silencing, but the silent state can be maintained only when the repressive modification signals are strong enough. With this assumption, we mean that DNA methylation can be induced when repressive histone modification is strong enough. This is consistent with the 


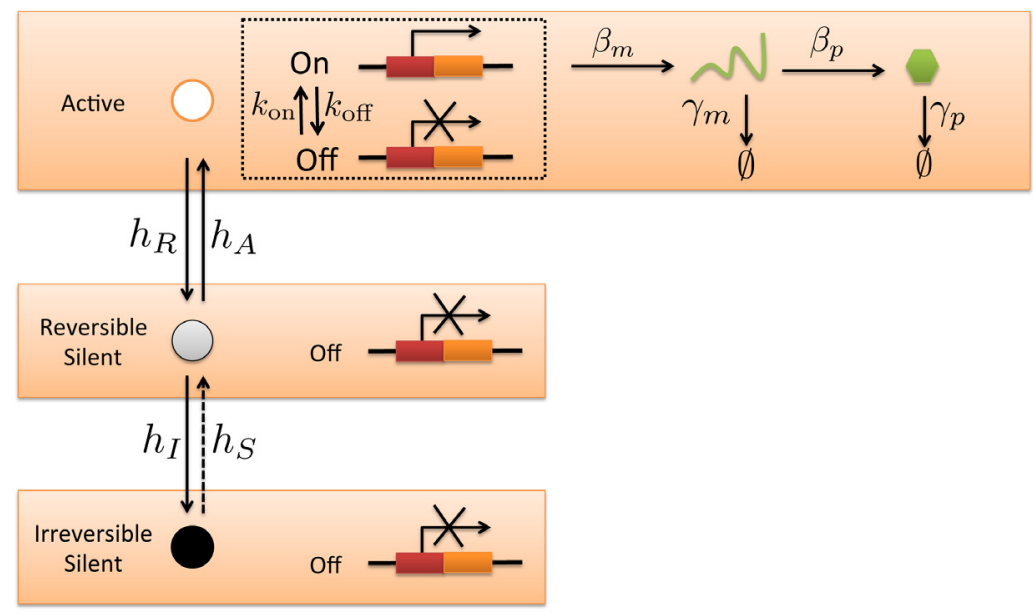

Fig. 2. (Color online) A three-state model of gene expression under epigenetic regulation. The chromatin state stochastically transits between actively expression (A), reversible silent (R), and irreversible silent (I) states. At the active chromatin state, RNAP repetitively binds to the promoter so that the promoter switches between $\mathrm{ON}$ and OFF state, and transcription is active at the ON promoter state. Replotted from Ref. 2.

collaboration between H3K9m2/3 and DNA methylation. $\frac{15}{}$ Hence, the silent state I is irreversible when the repressive modification is strong; however the transition from $\mathrm{I}$ to $\mathrm{R}$ is possible if the repressive modification is weakened. This leads to our modified three-state model (Fig. 2).

To connect nucleosome states with gene expression, we note that the states of nucleosomes can change the chromatin state to modulate gene expression. Here, we assumed that each nucleosome affects the rates of chromatin state transition by changing the energy barrier between different states. In particular, we assumed the following regulation of nucleosome states to the chromatin state transition: the bivalent state AR promotes the transition from active to reversible silent state, the modifications with active marks (AU or AA) induce activation of chromatin, and the modifications with repressive marks (UR or RR) tend to induce irreversible silence. Under these assumptions, let $f_{X}(X=U U, A U, U R, A A, A R, R R)$ be the fractions of nucleosomes at state $X$, respectively, and define $f_{A}=f_{A U}+f_{A A}$ and $f_{R}=$ $f_{U R}+f_{R R}$ as the tendency of active transcription and repressive transcription, respectively. The chromatin state transition rates can be expressed as functions of $f_{A}, f_{R}$ or $f_{A R}$, respectively.

The mechanisms of how histone modifications affect the chromatin state dynamics remain mostly unknown. In terms of mathematical formulation, while we assumed that the histone modifications can alter the chemical energy of chromatin structure dynamics, we introduced the following exponential functions for the dependence of chromatin state transition rates with the nucleosome states

$$
h_{R}=\bar{h}_{R} e^{-\mu_{R}\left(1-f_{A R}\right)},
$$




$$
\begin{aligned}
h_{A} & =\bar{h}_{A} e^{-\mu_{A}\left(1-f_{A}\right)}, \\
h_{I} & =\bar{h}_{I} e^{-\mu_{I}\left(1-f_{R}\right)} .
\end{aligned}
$$

The recovery from irreversible silent I to reversible silent $\mathrm{R}$ was assumed to be dependent on $f_{R}$ in a threshold manner

$$
h_{S}= \begin{cases}0, & f_{R} \geq 0.2, \\ \bar{h}_{S}, & f_{R}<0.2 .\end{cases}
$$

We referred to the standard model of gene expression for the process of transcription and translation. ${ }^{9,16}$ At the active expression state, RNA polymerase (RNAP) repeatedly binds to the promoter and triggers the transcription process. The promoter randomly switches between $\mathrm{ON}$ and $\mathrm{OFF}$ state with rates $k_{\mathrm{on}}$ and $k_{\mathrm{off}}$, respectively. mRNAs are synthesized at a rate $\beta_{m}$ when the promoter is ON, and degrades or dilutes at a rate $\gamma_{m}$. The translation and degradation/dilution rates of proteins are $\beta_{p}$ and $\gamma_{p}$, respectively.

\subsection{Enzyme activity and feedback regulation}

To consider the possibility of cell type transition during sequential cell divisions, we assumed a feedback loop of chromatin state transition in which the enzyme activities for the methylation/demethylation of $\mathrm{H} 3$ histone can be regulated by the gene expression production proteins (Fig. 3). Methylation/demethylation enzyme activities can be regulated in various ways, such as protein-protein interactions, cofactor availability and post-translational modifications. ${ }^{10}$ Usually, the gene production proteins can regulate enzyme activities either directly or indirectly. While we lump the intermediate steps between the gene product and enzyme activities, one can formulate the enzyme activities as a function of the protein number. Thus, let $P$ be the number of proteins, the enzyme activities of active marker writer $\left(W_{A}\right)$, repressive marker writer $\left(W_{R}\right)$, active marker eraser $\left(E_{A}\right)$ and repressive marker

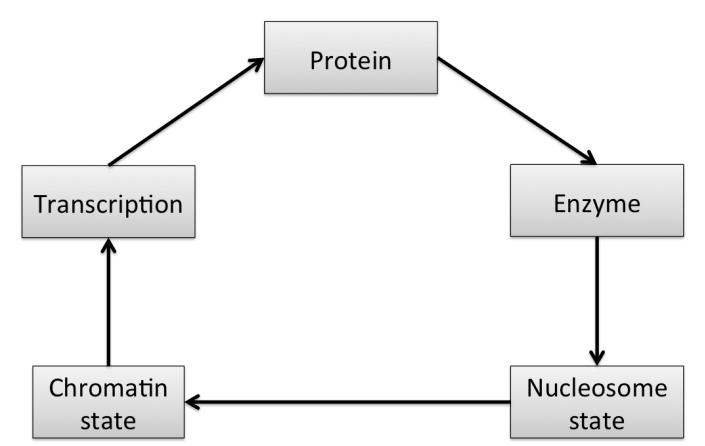

Fig. 3. The feedback loop of gene expression. Transcription production proteins regulate the enzyme activities to mediate nucleosome state and chromatin state transition. 
eraser $\left(E_{R}\right)$ are formulated as Hill type functions

$$
W_{X}=\bar{W}_{X} \frac{(P / V)^{m}}{K_{W}^{m}+(P / V)^{m}}, \quad E_{X}=\bar{E}_{X} \frac{K_{E}^{n}}{K_{E}^{n}+(P / V)^{n}}, \quad X=A, R,
$$

where $V$ represents the cell volume. Here, we have assumed that the proteins tend to activate writer enzymes and repress eraser enzymes. The methylation/demethylation rates $k_{X}$ and $j_{X}$ are dependent on the enzyme activities as

$$
k_{X}=\bar{k}_{X} \frac{W_{X}}{1+W_{X}}, \quad j_{X}=\bar{j}_{X} \frac{E_{X}}{1+E_{X}} .
$$

Here, we have normalized the EC50 of enzyme activities in each case as one.

Now, Eqs. (6) and (7) together introduce a feedback from protein production to nucleosome state transition dynamics. In the following discussions, to turn off the feedback regulations, we simply assumed that the enzyme activities are very high, so that $k_{X}=\bar{k}_{X}$ and $j_{X}=\bar{j}_{X}$.

\subsection{Numerical scheme}

We applied stochastic simulation algorithm (SSA) in accordance with the above chemical reactions. With this numerical scheme, stochasticities in histone modification, chromatin state transition, promoter activity, transcription and translation are involved in model simulation. To simulate the effects of many cell cycles, we took the length of one cell cycle as $24 \mathrm{~h}$. To consider cell division, we randomly assigned histone marks ( $A$ or $R$ at a histone) to one of the daughter cells and a newly synthesized histone (with state $U$ ) to the other daughter cell. The cell volume was taken as $V=1+t / 24(0 \leq t \leq 24 \mathrm{~h})$ within each cell cycle. In all simulations, the total number of nucleosomes is $N=60$.

\section{Results}

\subsection{Dynamics of the chromatin state}

Our model describes the stochastic chromatin dynamics that involve both stochastic kinetics in histone modifications and random partitions in cell division. In this model, the stochastic transitions between nucleosome states play important roles in the modulation of chromatin states, which in turn controls the efficiency of gene expression. First, we asked how the kinetics of nucleosome state transition may affect the stochastic dynamics of chromatin states. To this end, we first omitted the feedback regulations by taking $k_{X}=\bar{k}_{X}, j_{X}=\bar{j}_{X}$ in Eq. (7) and varied the rate constants that are involved in the nucleosome state transition [Fig. 1(B)] to investigate the dynamics of chromatin states. Hereafter, we used the fractions of each nucleosome state, $f_{A}, f_{R}$ and $f_{A R}$, to represent the epigenetic states of the gene.

In the simulation, there are sudden changes in the epigenetic states at the beginning of each cell cycle along with DNA replication, after which the epigenetic states quickly return to their stationary states at latter stages of a cell cycle [Fig. 4(A)]. 

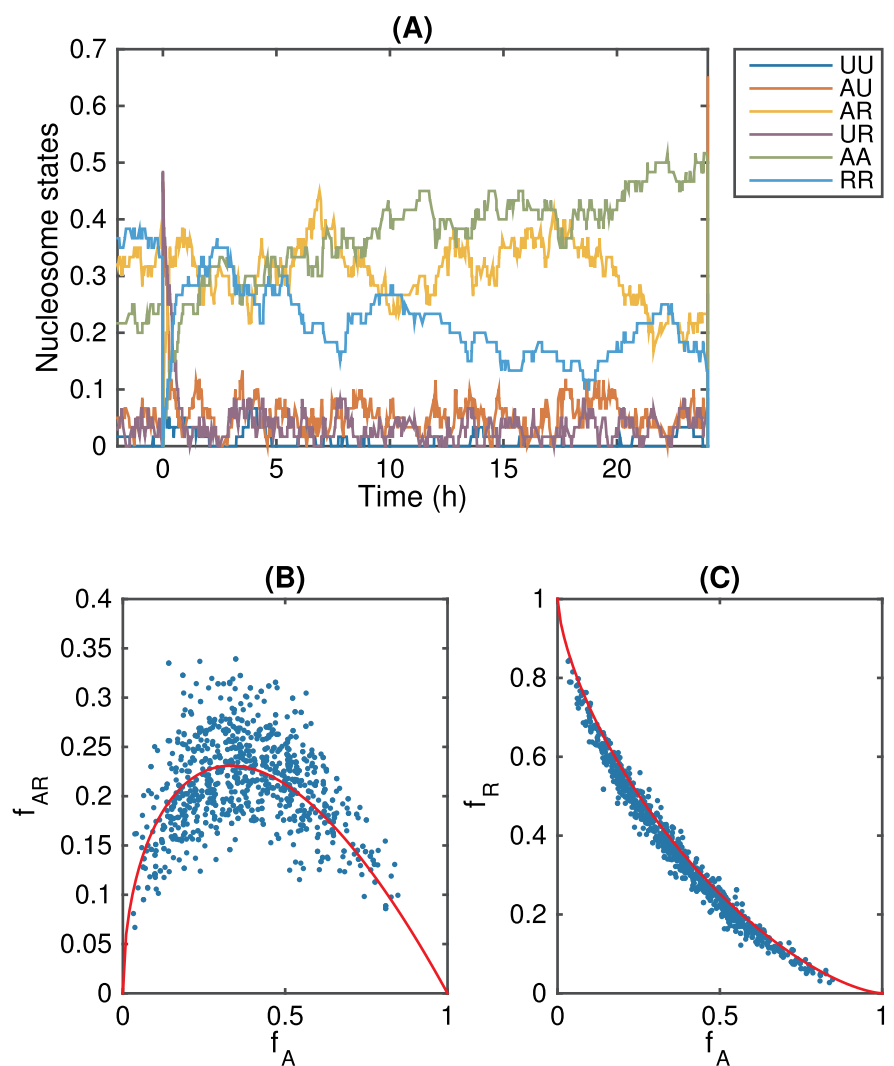

Fig. 4. (Color online) Nucleosome state dynamics. (A) A sample dynamics of nucleosome states in one cell cycle (starts at $0 \mathrm{~h}$ ). Curves show the fraction of nucleosome states at each state. (B) The average nucleosome state of bivalency $\left(f_{A R}\right)$ versus active transcription $\left(f_{A}\right)$. (C) The average nucleosome state of repressive transcription $\left(f_{R}\right)$ versus active transcription $\left(f_{A}\right)$. Dots show simulation results obtained from 1000 sets of randomly selected parameters over the region: $0.2 \leq m_{A}, m_{R} \leq 1.0,0.5 \leq \bar{k}_{A}, \bar{k}_{R} \leq 2.0$ and $0.2 \leq \bar{j}_{A}, \bar{j}_{R} \leq 1.0$. The feedback regulations were turned off in simulations $\left(\bar{k}_{X}=\bar{k}_{X}, \bar{j}_{X}=\bar{j}_{X}\right)$. The red solid curves show the fitting with Eq. (8).

Thus, we can represent the stationary state by the average levels of the epigenetic states at the later stages of a cell cycle; the stationary states are dependent on the chemical reaction rates defined in model simulations. To investigate the correlations between various epigenetic states, we randomly selected 1000 sets of parameters, and for each set of parameters we ran the simulation for 10 cell cycles; the average epigenetic states at the 10th cell cycle were obtained for each set of parameters. Despite the random parameter sets, we found obvious nonlinear correlations between the epigenetic states of bivalency $\left(f_{A R}\right)$ and the active transcription $\left(f_{A}\right)$, and between the states of repressive transcription $\left(f_{R}\right)$ and active transcription [Figs. 4(B) and 4(C)]:

$$
f_{A R}=\frac{3}{5} f_{A}^{1 / 2}\left(1-f_{A}\right), \quad\left(1-f_{R}\right)^{3 / 2}+\left(1-f_{A}\right)^{2 / 3}=1 .
$$


The correlation Eq. (8) suggests that despite the multidimensional epigenetic states, the tendency of active transcription can provide a rough description of the epigenetic state of the promoter. In particular, if $0.3<f_{A}<0.5$, we have $0.17<f_{A R}<$ 0.27 and $0.25<f_{R}<0.45$ for most randomly selected parameters. Hence, in the following discussion of bivalent epigenetic states, we selected parameters from this region, and used $f_{A}$ to represent the epigenetic state.

\subsection{Stochasticity in gene expression}

To study how random kinetics of bivalent histone modifications affect the stochasticity in gene expression, we choose a set of parameters so that the probability of stationary bivalency is large $\left(f_{A R}>0.25\right)$. In this case, the epigenetic states $f_{A}, f_{R}$ and $f_{A R}$ fluctuate around levels from 0.2 to 0.6 [Fig. 5(A)]. The transition rates of

(A)

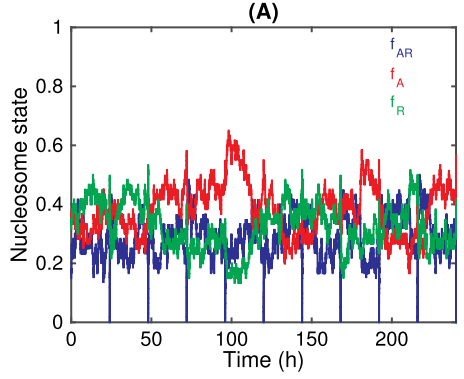

(C)

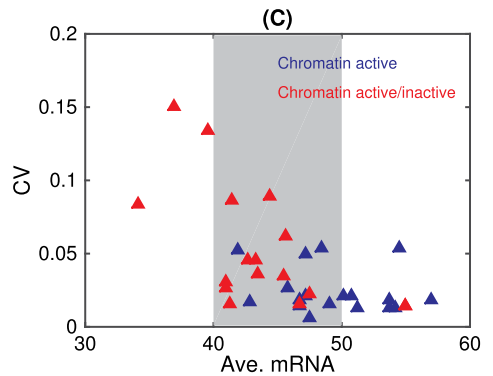

(B)

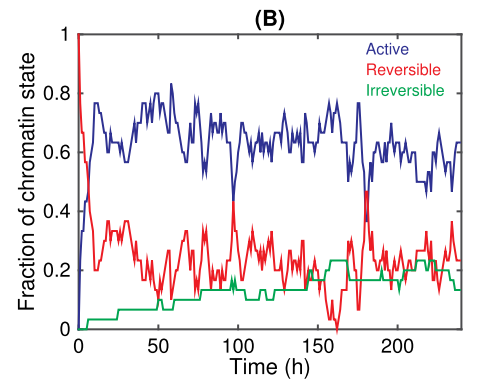

(D)

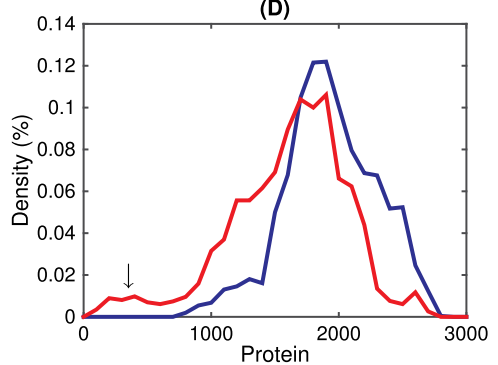

Fig. 5. (Color online) Chromatin state dynamics and stochasticity in gene expression. (A) A sample dynamics of the average nucleosome state. (B) Dynamics of the fraction of chromatin state over 30 cells. In simulations, the feedback regulations are turned off, and parameters for the kinetics of histone modification and chromatin state transitions are: $m_{A}=0.96 \mathrm{~h}^{-1}, m_{R}=0.31 \mathrm{~h}^{-1}, \bar{k}_{A}=$ $0.93 \mathrm{~h}^{-1}, \bar{k}_{R}=1.69 \mathrm{~h}^{-1}, \bar{j}_{A}=0.65 \mathrm{~h}^{-1}, \bar{j}_{R}=0.21 \mathrm{~h}^{-1}, \bar{h}_{R}=0.29 \mathrm{~h}^{-1}, \bar{h}_{A}=0.35 \mathrm{~h}^{-1}, \bar{h}_{I}=$ $0.04 \mathrm{~h}^{-1}, \bar{h}_{S}=2.0 \mathrm{~h}^{-1}, \mu_{R}=2.0 \mathrm{~h}^{-1}, \mu_{A}=1.0 \mathrm{~h}^{-1}, \mu_{I}=2.0 \mathrm{~h}^{-1}$. Here, the parameter values refer to the order of rates in Refs. 12 and 28. (C) Relations between the coefficient of variance (CV) and average mRNA levels for the two models: chromatin state kept active (blue), or chromatin state regulated by the nucleosome state kinetics (red). (D) Probability of protein products of the two models: chromatin state kept active (blue), and nucleosome-mediated chromatin activity (red). Parameters for the transcription dynamics are: $k_{\mathrm{on}}=4 \mathrm{~h}^{-1}$ (chromatin state kept active) or $k_{\mathrm{on}}=8 \mathrm{~h}^{-1}$ (nucleosome-mediated chromatin activity), $k_{\mathrm{off}}=1 \mathrm{~h}^{-1}, \beta_{M}=2 \mathrm{~h}^{-1}, \gamma_{M}=$ $0.03 \mathrm{~h}^{-1}, \beta_{P}=2 \mathrm{~h}^{-1}, \gamma_{P}=0.05 \mathrm{~h}^{-1}$. Other parameters of the nucleosome state kinetics and chromatin state regulation were the same as those used in $(\mathrm{A})$ and $(\mathrm{B})$; in the model chromatin kept active, we set $h_{R}=h_{I}=0$ to block the nucleosome-mediated chromatin inactivation. 
chromatin state are determined by the average levels of modified nucleosomes according to Eqs. (2)-(5). At each cell cycle, the nucleosome states quickly recover to their stationary state after DNA replication, and the dynamics of chromatin state transition are slow processes; the reassignment of nucleosome state at DNA replication did not obviously affect the chromatin state dynamics [Fig. 5(B)]. Thus, the stationary fractions of the three chromatin states (active transcription, reversible silent or irreversible silent) are dependent on the histone modification dynamics and the chromatin state transition rates. Figures $5(\mathrm{~A})$ and $5(\mathrm{~B})$ shows the simulation dynamics of the fractions of each chromatin state over a period of 10 cell cycles.

To investigate how chromatin dynamics affect the stochasticity in gene expression, we compared the fluctuation of mRNA levels obtained from two different models: either the chromatin state was kept active (accordingly, $h_{R}=h_{I}=0$ ), or the chromatin state was regulated by the histone kinetics. The fluctuation was represented by the coefficient of variance $(\mathrm{CV})$, which is the variance divided by the square of the mean. Usually, the presence of nucleosome mediated the chromatin's active/inactive state and can attenuate the transcription activity. Hence, in simulation, we adjusted the transcription rate in the two models so that they gave the same levels of average mRNA productions (Fig. 5). Results show that including the layer of nucleosome-mediated chromatin inactivity to transcription dynamics obviously upregulated the CV [Fig. 5(C), gray area]. In Appendix A, we show that the $\mathrm{CV}$ of mRNA products is given by

$$
\eta_{2}^{2}=\frac{1}{\left\langle X_{2}\right\rangle}+\frac{1-\frac{\theta}{1+\tau_{0} / \tau_{2}}}{1-\theta} \eta_{12}+\left(1-\frac{1}{1+\tau_{0} / \tau_{2}}\right) \eta_{0}^{2} .
$$

Here, the parameter $\theta>0$ represents the relative rate of chromatin silencing. From Eq. (9), it is obvious that $\partial \eta_{2}^{2} / \partial \theta>0$, which provides an analytic validation of the increasing CV under nucleosome-mediated chromatin activity. As a direct consequence, the larger fluctuations in mRNA numbers imply the wider range distribution of protein products in the presence of nucleosome-mediated chromatin activity [Fig. 5(D)]. In particular, the protein number can be very low due to the nucleosome-regulated inactivation of the chromatin state [Fig. 5(D), arrow].

\subsection{Bimodal transcription dynamics under a feedback regulation to the transition of nucleosome states}

To examine how the positive feedback to histone modifications affects gene expression, we assumed that a positive feedback of gene expression is formed while protein products promote the writer of active modification and inhibit the eraser of active modification. In this case, we have

$$
W_{A}=\bar{W}_{A} \frac{(P / V)^{m}}{K_{W}^{m}+(P / V)^{m}}, \quad k_{A}=\bar{k}_{A} \frac{W_{A}}{1+W_{A}},
$$


and

$$
E_{A}=\bar{E}_{A} \frac{K_{E}^{n}}{K_{E}^{n}+(P / V)^{n}}, \quad j_{A}=\bar{j}_{A} \frac{E_{A}}{1+E_{A}}
$$

from Eqs. (6) and (7). Moreover, there is no feedback to the repressive marker enzymes so that $W_{R}$ and $E_{R}$ are constants, and

$$
k_{R}=\bar{k}_{R} \frac{W_{R}}{1+W_{R}}, \quad j_{R}=\bar{j}_{R} \frac{E_{R}}{1+E_{R}} .
$$

First, we randomly took $0<\bar{k}_{A}<1$ and $0<\bar{j}_{A}<2$ and run the model simulation over 10 cell cycles until the nucleosome state reaches the stationary state. The nucleosome states are represented by the fractions $f_{A R}$ and $f_{A}$, which show similar dependence as in the case without feedback regulation given by Eq. (8) [Fig. 6(A)]. Next, we divided the parameter regions in the $\left(\bar{k}_{A}, \bar{j}_{A}\right)$ plane into three
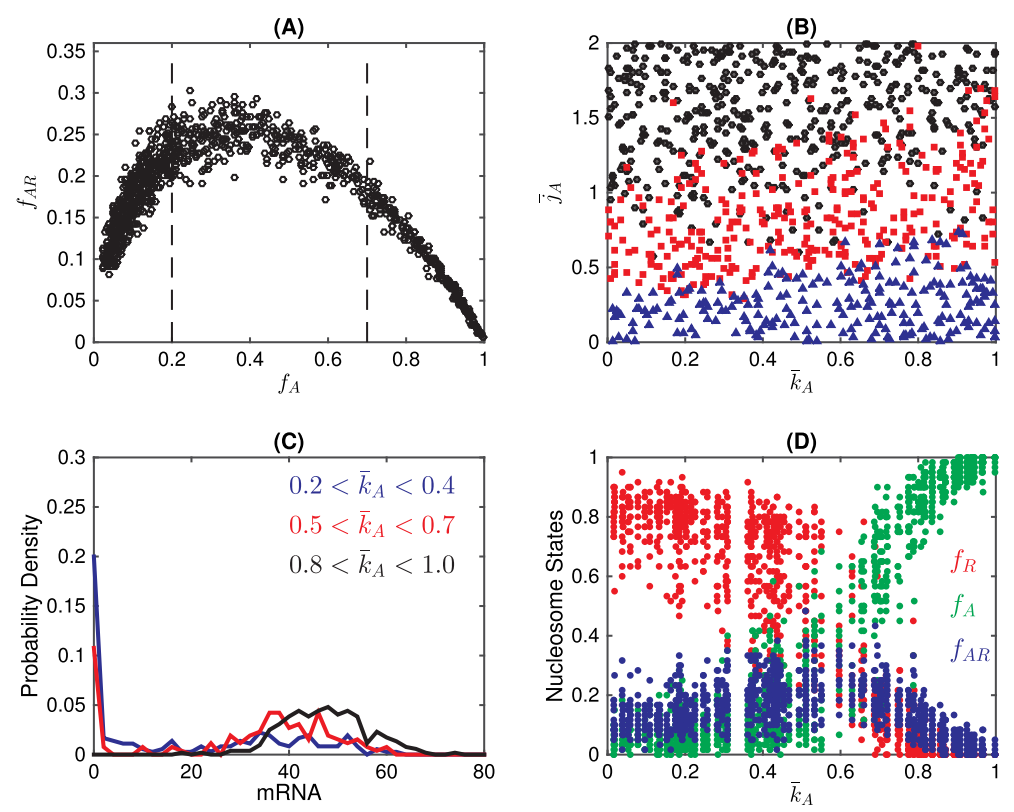

Fig. 6. (Color online) Bimodal distribution of nucleosome states. (A) The nucleosome states $f_{A R}$ vs. $f_{A}$ over 1000 sample simulations with randomly selected parameters $0<\bar{k}_{A}<1$ and $0<\bar{j}_{A}<2$. (B) Parameters $\bar{k}_{A}$ and $\bar{j}_{A}$ used in (A), all parameters are separated into three groups according to $f_{A}$ (dashed lines in (A)): $f_{A}<0.2$ (black dots), $0.2 \leq f_{A}<0.7$ (red starts) and $f_{A} \geq 0.7$ (blue triangles). (C) mRNA level at stationary state for different values of $\bar{k}_{A}$ (here $\bar{j}_{A}=2\left(1-\bar{k}_{A}\right)$. (D) Nucleosome states $\left(f_{R}(\right.$ red $), f_{A}$ (blue) and $f_{A R}$ (black)) for different values of $\bar{k}_{A}$. Simulation protocol is the same as in (C). All simulations are run for 10 cell cycles, and the results at the last cycle are collected. Here, parameters are: $\bar{W}_{A}=\bar{E}_{A}=2, K_{W}=K_{E}=1000$, $m=n=2$, and $k_{A}$ and $j_{A}$ are given by Eqs. (10) and (11); there is no feedback to the repressive maker enzymes so that $W_{R}=E_{R}=2, \bar{k}_{R}=2.5 \mathrm{~h}^{-1}, \overline{j_{R}}=0.5 \mathrm{~h}^{-1}$, and $k_{R}$ and $j_{R}$ are given by Eq. (12); other parameters referred to those used in Fig. 5 with nucleosome-mediated chromatin activity. 
parts according to the separation $f_{A}=0.2$ and $f_{A}=0.7$ [Fig. 6(B)], and found that the nucleosome state is mainly dependent on the eraser enzyme activity $\bar{j}_{A}$.

Next, we set $\bar{j}_{A}=2\left(1-\bar{k}_{A}\right)$ and $0<\bar{k}_{A}<1$ to examine how the transcription dynamics changes with nucleosome state transition in the presence of feedback regulation. The mRNA level can display bimodal distribution for small $\bar{k}_{A}$, or monomodal distribution with higher level transcription when $\bar{k}_{A}$ is large [Fig. 6(C)]. Figure $6(\mathrm{D})$ shows the dependence of nucleosome states $\left(f_{R}, f_{A}\right.$ and $\left.f_{A R}\right)$ on the parameter $\bar{k}_{A}$. The fraction of repressive marks $f_{R}$ decreases with $\bar{k}_{A}$, and the fraction of active marks $f_{A}$ increases with $\bar{k}_{A}$. Moreover, there is a transition at about $0.5<\bar{k}_{A}<0.7$, at which the fraction of bivalent marks $f_{A R}$ reaches a relatively high level of about 0.3 . Thus, the bivalent histone modification is possible when the enzyme activities take proper values.

We selected sample trajectories with $\bar{k}_{A}=0.6,0.7,0.8$, respectively; dynamics of the nucleosome states are shown in Fig. 7. In these three specific cases, the bivalent levels $f_{A R}$ are similar; however, the dynamics of the numbers of active and repressive marks are different. In particular, there are obvious switches from high to low-level active markers when $\bar{k}_{A}=0.6$ and $\bar{k}_{A}=0.7$. According to the model in Fig. 2, the chromatin can be trapped to the irreversible silent state when $f_{R}>0.2$. Hence, when repressive modifications become dominant (small $\bar{k}_{A}$ in model simulations), the gene can become silent in long-run dynamics. The switch from active transcription to silence was also confirmed by the mRNA dynamics
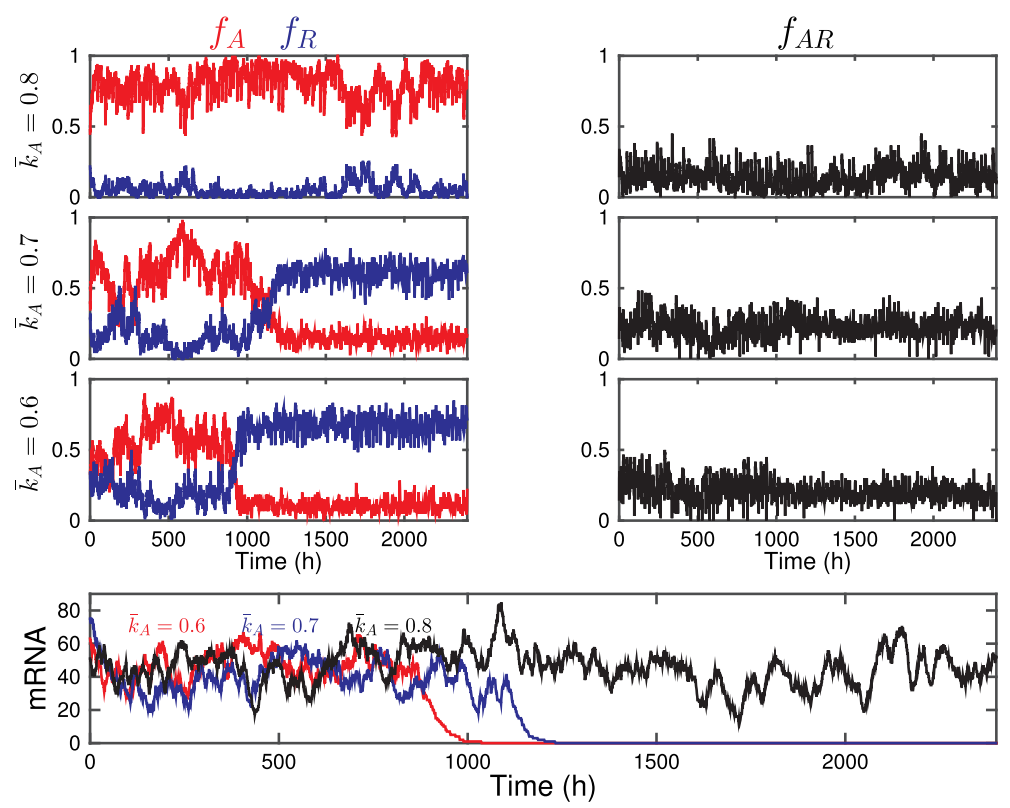

Fig. 7. (Color online) Dynamics of nucleosome state. Example dynamics of the nucleosome states $\left(f_{A}, f_{R}\right.$ and $f_{A R}$, upper panels) and mRNA (bottom panel) for values $\bar{k}_{A}=0.6,0.7,0.8\left(\mathrm{~h}^{-1}\right)$, respectively. Data obtained from the sample trajectory in Fig. 6(C) and 6(D). 
(Fig. 7, bottom panel). We note that in model simulation there is no external noise perturbation, and hence the switches are induced merely by intrinsic noise. In particular, the stochastic kinetics of nucleosome states transition play important roles in regulating gene transcription.

\subsection{Effects of the feedback strength}

In the regulatory circuit shown by Fig. 3, the feedback strength was represented with the parameters of EC50 $K_{W}$ and $K_{E}$ in Eqs. (10) and (11), which control the efficiency of proteins to the activation of writer enzymes and the repression of eraser enzymes. Smaller values of $K_{W}$ and $K_{E}$ yield more significant feedback regulations. To investigate the effects of the feedback strengths, we took $K_{E}=K_{W}$ and varied $K_{W}$ over the range from 0 to 2000. For each value of $K_{W}$, we repeated the simulation for 10 independent runs, each for 10 cycles. The final state of nucleosome activity $f_{A}$ at each run is shown in Fig. 8(A). The feedback strength can be divided into three regions. When $K_{W}$ is small $\left(K_{W} \leq 300\right)$, the nucleosome activities are high $\left(f_{A} \approx 1\right)$; when $K_{W}$ took intermediate values $\left(300<K_{W}<1000\right)$, the nucleosome activities displayed bimodal distributions, either intermediate activities or low-level activities $\left(f_{A}<0.2\right)$; and when $K_{W}$ further increased, the intermediate activities decreased toward the lower level values. The probability density
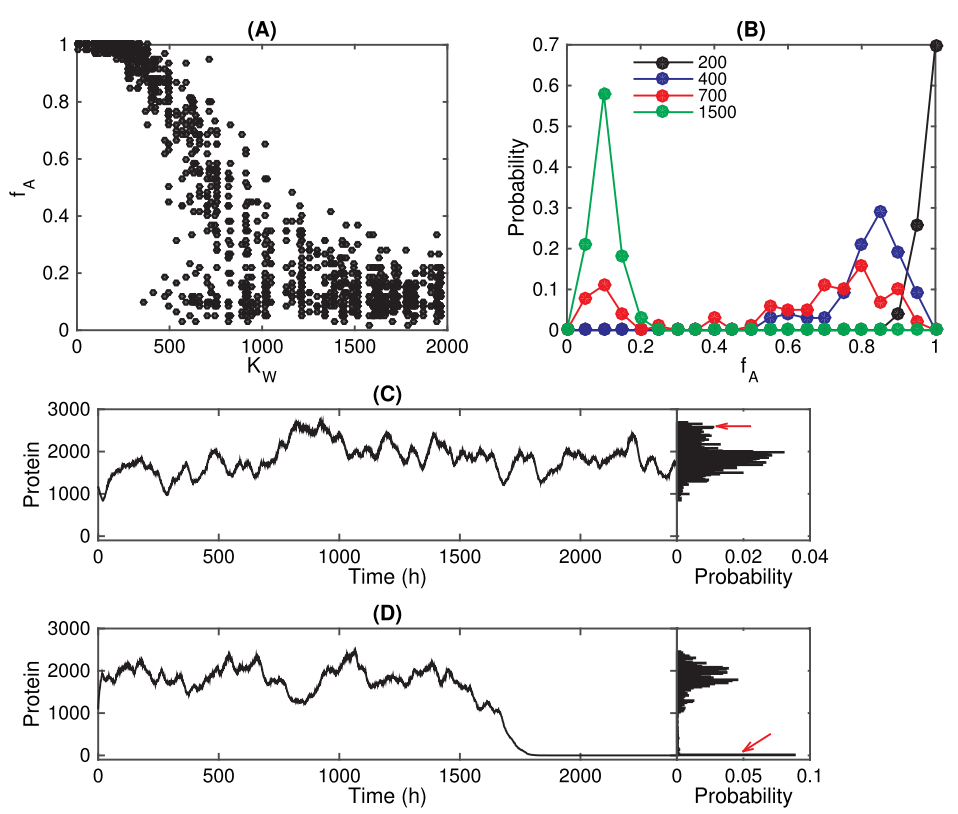

Fig. 8. (Color online) Effects of feedback strengths. (A) Dependence of the activity nucleosome state $f_{A}$ with the feedback strength $K_{W}$ (here $K_{E}=K_{W}$ ). (B) Probability density of $f_{A}$ versus the feedback strength $K_{W}$. (C)-(D) Sample trajectories of protein production, with $K_{W}=700$, and the corresponding probability densities. Red arrows show the high-level expression (C) and gene silence $(\mathrm{D})$, respectively. 
of the nucleosome activity $f_{A}$ at different values of $K_{W}$ also confirmed the above observations [Fig. 8(B)]. When $K_{W}=200$, the probability density has a single peak at $f_{A}=1$, and when $K_{W}=700$, the probability density has two peaks, at $f_{A}=0.8$ and $f_{A}=0.1$, respectively. Figures $8(\mathrm{C})$ and $8(\mathrm{D})$ show two example trajectories for the case $K_{W}=700$. In these two trajectories, the protein numbers obviously fluctuated during time evolution, and displayed either high level expression [Fig. 8(C)], or zero products due to the irreversible silencing of chromatin state [Fig. $8(\bar{D})$ ].

\subsection{Nucleosome state transition between cell cycles}

Histone modification can be inherited a cross the cell cycle, hence the transition of nucleosome states during cell regeneration is crucial in the study of tissue development and cell heterogeneity. To quantify the nucleosome transition cross cell cycle, we defined the nucleosome state at each cycle by the value of nucleosome activity, $f_{A}$, and found the iterative map of $f_{A}$ from one cycle to the next [Fig. 9(A)]. This map clearly shows that in each cell division, the nucleosome state after cell division (the daughter cell) is obviously correlated with the state prior to cell division (the mother cell). In the current model, because of the bimodal distribution of nucleosome states due to the positive feedback, the iterative map can be separated into two parts. In the lower active region, the average activities of the daughter cell are insensitive with that of the mother cell, and in the higher active region the average activities of the daughter cell are positively correlated with the mother cell [Fig. 9(B)]. Moreover, the probability density of $f_{A}$ for the daughter cells, given the state of the mother cell, can be well-formulated as binomial distribution, so that

$$
\begin{aligned}
P\left(f_{A, n+1}=f \mid f_{A, n}=g\right) & =N C_{N}^{\lfloor f N\rfloor} p^{\lfloor f N\rfloor}(1-p)^{N-\lfloor f N\rfloor}, \quad \text { where } \\
p & =\left.\left\langle f_{A, n+1}\right\rangle\right|_{f_{A, n}=g} .
\end{aligned}
$$
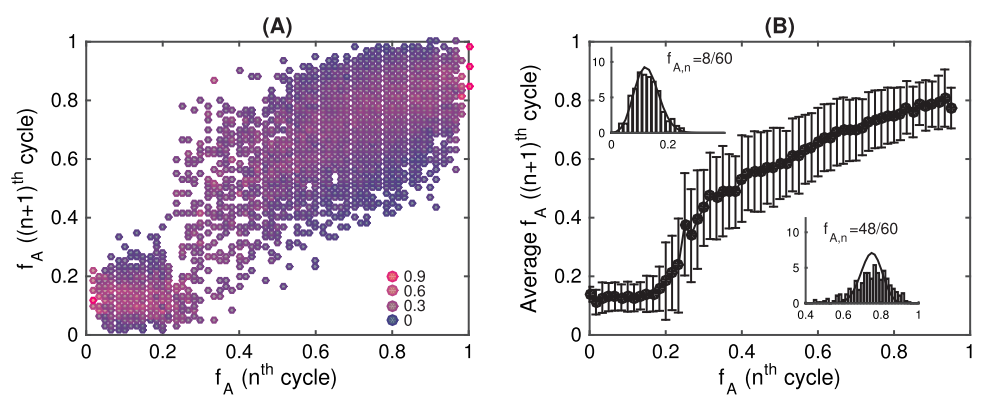

Fig. 9. (Color online) Nucleosome state transition cross cell cycles. (A) Diagram of the iterative map for the nucleosome activities $f_{A}$ a cross sequential cell cycles (from $n$th to the $(n+1)$ th cycles). Color of each dot represents the probability at the $(n+1)$ th cycle, given the $f_{A}$ value at the $n$th cycle. (B) Mean (black dots) and standard deviation (error bars) of $f_{A}$ at the $(n+1)$ th given the value at the $n$th cycle. Insets are probability density of the two situations, $f_{A}=48 / 60$ and $f_{A}=8 / 60$, at the $n$ th, respectively. Bars from simulation data, and solid lines are fitting with binomial distribution Eq. (13). Here, $K_{W}=700$. 

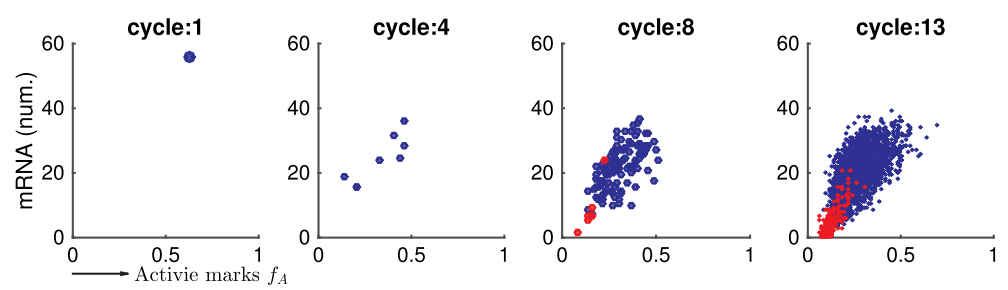

Fig. 10. (Color online) Spontaneous cell state transition. Simulation of a clone from a single cell over 13 cell cycles. Dots show the mRNA number and active marks $f_{A}$ of each single cell at different cycles. Red dots specify the differentiated cells (irreversible silent chromatin).

Here, $f_{A, n}$ means the value $f_{A}$ at the $n$th cycle. Figure $9(\mathrm{~B})$ shows that the binomial distribution fits well with simulation data.

The random transition dynamics in Fig. 9 implies a possibility that the nucleosome state of a cell can spontaneously transit to another state following a sequence of cell divisions. To investigate how dynamics of gene expression in a cell lineage evolved following sequential cell divisions, we started from a single cell and performed model simulation to obtain the dynamics of gene expression and nucleosome state of the generated cell clone at the single-cell level. In simulation, to mimic cell division, we halve the numbers of mRNA and protein, as well as the cell volume $V$, and randomly assign the modified histone marks to the daughter cells. Figure 10 shows the transition of mRNA level and active marks $f_{A}$ from cycle 1 to cycle 13. Here, we mark the dots in red for those cells in which the chromatin state of the gene is irreversible silent; representing the spontaneous transition from active transcription to silencing following cell division. This result shows that the present model of random dynamics in histone modification is capable of silencing the transcription activity of a gene following a sequential cell division.

\section{Discussion}

This study developed a computational model of gene expression with a positive feedback through the regulation of nucleosome state transitions. The nucleosome states were considered as bivalent histone modifications at the gene promoter. The model is a hybrid of different scale kinetics processes, including dynamics of histone modifications, three-stage chromatin states transition, gene expression, feedback regulation from protein products to enzyme activities, and the random partition of modification marks in cell division. In the model, two histone methylations, H3K4me3 which activates transcription, and H3K27me3, which represses transcription, were considered. Model simulations reveal that random dynamics of histone methylation can introduce an additional layer of stochasticity in gene expression, which is crucial when the chromatin dynamics become slow.

Histone modification has features of both inheritance and plasticity during cell division, which are essential for cell memory and differentiation. ${ }^{5,7,18,23}$ In our model, we considered the transition of nucleosome state during cell division as 
a random dynamics from mother cell to daughter cells. The random dynamics is well described by a sequence of conditional binomial distribution (Fig. 9). From our formulation, inheritance and plasticity of the nucleosome state can be quantified by the dependence of mean values of the daughter cells on that of the mother cells upon cell division [Eq. (13)]. Moreover, the plasticity of nucleosome dynamics suggests a possible spontaneous cell fate transition during stem cell regeneration. Many experiments have reported the changes in histone modification during embryo development and stem cell differentiation. $\frac{1,3,8,14,27}{}$ Moreover, single-cell RNAsequencing shows a continuous spectrum of stem cell differentiation. $\frac{17}{}$ Our model simulations reveal that, in the long-run cell regeneration, random dynamics in nucleosome transition can induce spontaneous silencing in the chromatin state and continuous mRNA dynamics in spite of the bimodal gene expression. These results may provide insights to the future study of how inheritance and plasticity of epigenetic regulation can work to regulate cell fate decision during development.

In addition to histone modification, many other effects can also affect the stochasticity in gene expression, such as the random binding of TFs, multisteps $\mathrm{ON}$ and OFF dynamics of the promoter, elongation dynamics of the RNAP, kinetics of DNA methylation, interfering with enhancers, etc. These interactions together lead to a complex dynamical process of gene expression, which is important for our understanding of the heterogeneity in multiple cell systems. ${ }^{21}$ Currently, various computational models have been developed trying to understand the stochasticity of gene expression based on different regulations; however, a comprehensive understanding of the long-term dynamics and cell-to-cell variability remain unclear, requiring more integrative models for the discovery of various time scale interactions.

\section{Acknowledgments}

This work was supported by the National Natural Science Foundation of China (Grant Nos. 914030101 and 11272169).

\section{Appendix A. Coefficient of Variance}

To obtain the CV of mRNA products when the chromatin dynamics are included in transcription regulation, we considered the model shown in Fig. 2. To simplify the calculations below, we omitted the transition of chromatin state from reversible silent to irreversible silent. We omitted the translation as well because we only calculate the CV of mRNA products. Hence, the system can be described by $\mathbf{X}=$ $\left(X_{0}, X_{1}, X_{2}\right)$, with $X_{0}$ the chromatin state (active, $X_{0}=1$, or reversible silent, $X=0), X_{1}$ the promoter state (ON, $X_{1}=1$, or OFF, $\left.X_{1}=0\right)$, and $X_{2}$ the number of mRNA products. The propensity functions and state-changed vectors of the reactions are given in Table A.1.

Let $P\left(X_{0}, X_{1}, X_{2}, t\right)$ be the probability density function of the system state, and

$$
P_{0}\left(X_{1}, X_{2}, t\right)=P\left(0, X_{1}, X_{2}, t\right), \quad P_{1}\left(X_{1}, X_{2}, t\right)=P\left(1, X_{1}, X_{2}, t\right) .
$$


Table A.1. The propensity functions $a_{j}(X)$ and state change vectors $\nu_{j}$ of the transcription dynamics. Here, the reaction channels 1 and 2 are for chromatin state transition, channels 3 and 4 are for the promoter state transition, channel 5 is for transcription, and channel 6 is for mRNA degradation/dilution.

\begin{tabular}{|c|c|c|}
\hline$j$ & $a_{j}(\mathbf{X})$ & $\nu_{j}$ \\
\hline 1 & $h_{R} X_{0}$ & $(-1,0,0)$ \\
\hline 2 & $h_{A}\left(1-X_{0}\right)$ & $(1,0,0)$ \\
\hline 3 & $\begin{cases}k_{\mathrm{off}} X_{1}, & X_{0}=1 \\
0, & X_{1}=0\end{cases}$ & $(0,-1,0)$ \\
\hline 4 & $\begin{cases}k_{\mathrm{on}}\left(1-X_{1}\right), & X_{0}=1 \\
0, & X_{1}=0\end{cases}$ & $(0,1,0)$ \\
\hline 5 & $\begin{cases}\beta_{m} X_{1}, & X_{0}=1 \\
0, & X_{1}=0\end{cases}$ & $(0,0,+1)$ \\
\hline 6 & $\gamma_{m} X_{2}$ & $(0,0,-1)$ \\
\hline
\end{tabular}

The chemical master equation (CME) of the system is formulated as

$$
\begin{aligned}
\frac{\partial P_{0}}{\partial t}\left(X_{1}, X_{2}, t\right)= & h_{R} P_{1}\left(X_{1}, X_{2}, t\right)-h_{A} P_{0}\left(X_{1}, X_{2}, t\right) \\
& +\gamma_{m}\left(X_{2}+1\right) P_{0}\left(X_{1}, X_{2}+1, t\right)-\gamma_{m} X_{2} P_{0}\left(X_{1}, X_{2}, t\right), \\
\frac{\partial P_{1}}{\partial t}\left(X_{1}, X_{2}, t\right)= & h_{A} P_{0}\left(X_{1}, X_{2}, t\right)-h_{R} P_{1}\left(X_{1}, X_{2}, t\right) \\
& +k_{\text {off }}\left(X_{1}+1\right) P_{1}\left(X_{1}+1, X_{2}, t\right) \\
& -k_{\text {off }} X_{1} P_{1}\left(X_{1}, X_{2}, t\right)+k_{\text {on }}\left(1-\left(X_{1}-1\right)\right) P_{1}\left(X_{1}-1, X_{2}, t\right) \\
& -k_{\text {on }}\left(1-X_{1}\right) P_{1}\left(X_{1}, X_{2}, t\right)+\beta_{m} X_{1} P_{1}\left(X_{1}, X_{2}-1, t\right) \\
& -\beta_{m} X_{1} P_{1}\left(X_{1}, X_{2}, t\right)+\gamma_{m}\left(X_{2}+1\right) P_{1}\left(X_{1}, X_{2}+1, t\right) \\
& -\gamma_{m} X_{2} P_{1}\left(X_{1}, X_{2}, X_{4}, t\right) .
\end{aligned}
$$

The average values of $X_{i}$ are defined as

$$
\left\langle X_{i}\right\rangle=\sum_{\mathbf{X}} X_{i} P(\mathbf{X}, t), \quad i=0,1,2 .
$$

Therefore, let

$$
\left\langle X_{i}\right\rangle_{k}=\sum_{X_{1}, X_{2}} X_{i} P_{k}\left(X_{1}, X_{2}, t\right), \quad k=0,1
$$

we have

$$
\left\langle X_{0}\right\rangle=\sum_{X_{1}, X_{2}} P_{1}\left(X_{1}, X_{2}, t\right)=\left\langle X_{0}\right\rangle_{1},
$$




$$
\begin{aligned}
\left\langle X_{i}\right\rangle & =\sum_{X_{1}, X_{2}} X_{i} P_{0}\left(X_{1}, X_{2}, t\right)+\sum_{X_{1}, X_{2}} X_{i} P_{1}\left(X_{1}, X_{2}, t\right) \\
& =\left\langle X_{i}\right\rangle_{0}+\left\langle X_{i}\right\rangle_{1}, \quad i=1,2 .
\end{aligned}
$$

From the above CME, we obtain

$$
\begin{aligned}
& \frac{d\left\langle X_{0}\right\rangle_{1}}{d t}=-\left(h_{R}\left\langle X_{0}\right\rangle_{1}-h_{A}\left(1-\left\langle X_{0}\right\rangle_{1}\right)\right), \quad \frac{d\left\langle X_{1}\right\rangle_{0}}{d t}=h_{R}\left\langle X_{1}\right\rangle_{1}-h_{A}\left\langle X_{1}\right\rangle_{0}, \\
& \frac{d\left\langle X_{1}\right\rangle}{d t}=-k_{\mathrm{off}}\left\langle X_{1}\right\rangle_{1}+k_{\mathrm{on}}\left(\left\langle X_{0}\right\rangle-\left\langle X_{1}\right\rangle_{1}\right), \quad \frac{d\left\langle X_{2}\right\rangle}{d t}=-\gamma_{m}\left\langle X_{2}\right\rangle+\beta_{m}\left\langle X_{1}\right\rangle_{1} .
\end{aligned}
$$

Thus, the average values at stationary state are

$$
\begin{aligned}
\left\langle X_{0}\right\rangle & =\frac{h_{A}}{h_{R}+h_{A}}, \\
\left\langle X_{1}\right\rangle_{1} & =\frac{h_{A}}{h_{R}+h_{A}}\left\langle X_{1}\right\rangle=\left\langle X_{1}\right\rangle\left\langle X_{0}\right\rangle \\
\left\langle X_{1}\right\rangle & =\frac{k_{\mathrm{on}}}{k_{\mathrm{on}}+k_{\mathrm{off}}} \\
\left\langle X_{2}\right\rangle & =\frac{\beta_{m}}{\gamma_{m}}\left\langle X_{1}\right\rangle\left\langle X_{0}\right\rangle
\end{aligned}
$$

Now, considered the variance

$$
\sigma_{i k}=\left\langle\left(X_{i}-\left\langle X_{i}\right\rangle\right)\left(X_{k}-\left\langle X_{k}\right\rangle\right)\right\rangle=\sum_{\mathbf{x}}\left(x_{i}-\left\langle X_{i}\right\rangle\right)\left(x_{k}-\left\langle X_{k}\right\rangle\right) P(\mathbf{x}, t) .
$$

Thus, the CME yields

$$
\frac{d \sigma_{i k}}{d t}=\left\langle A_{i}(X)\left(X_{k}-\left\langle X_{k}\right\rangle\right)\right\rangle+\left\langle A_{k}(X)\left(X_{i}-\left\langle X_{k}\right\rangle\right)\right\rangle+\left\langle B_{i k}(X)\right\rangle,
$$

where

$$
A_{i}(X)=\sum_{j} v_{j i} a_{j}(X), \quad B_{i k}=\sum_{j} v_{j i} v_{j k} a_{j}(X) .
$$

A tedious calculation shows

$$
\begin{aligned}
& \left\langle A_{1}(X)\left(X_{i}-\left\langle X_{i}\right\rangle\right)\right\rangle=-\left(h_{A}+h_{R}\right) \sigma_{1 i} \\
& \left\langle A_{2}(X)\left(X_{i}-\left\langle X_{i}\right\rangle\right)\right\rangle=-\left(k_{\text {on }}+k_{\text {off }}\right)\left(\sigma_{2 i}\right)_{1}, \\
& \left.\left.\left\langle A_{3}(X)\left(X_{i}-\left\langle X_{i}\right\rangle\right)\right\rangle=\beta_{m}\left(\sigma_{2 i}\right)_{1}+\beta_{m}\left\langle X_{2}\right\rangle\left\langle X_{i}\right\rangle-\left\langle X_{i}\right\rangle\right)\right\rangle_{1}-\gamma_{m} \sigma_{3 i} .
\end{aligned}
$$

Since

$$
\begin{aligned}
& \left\langle X_{0}-\left\langle X_{0}\right\rangle\right\rangle_{1}=\left\langle X_{0}\right\rangle\left(1-\left\langle X_{0}\right\rangle\right) \\
& \left\langle X_{1}-\left\langle X_{1}\right\rangle\right\rangle_{1}=\left\langle X_{1}\right\rangle_{1}-\left\langle X_{1}\right\rangle\left\langle X_{0}\right\rangle=0, \\
& \left\langle X_{2}-\left\langle X_{2}\right\rangle\right\rangle_{1}=\left\langle X_{2}\right\rangle_{1}-\left\langle X_{2}\right\rangle\left\langle X_{0}\right\rangle
\end{aligned}
$$


and

$$
\left(\sigma_{21}\right)_{1}=\sigma_{21}, \quad\left(\sigma_{22}\right)_{1}=\left\langle X_{1}\right\rangle \sigma_{22}, \quad\left(\sigma_{23}\right)_{1}=\frac{h_{A}+\gamma_{m}}{h_{A}+h_{R}+\gamma_{m}} \sigma_{23} .
$$

We have

$$
\begin{aligned}
\left\langle A_{0}(x)\left(X_{0}-\left\langle X_{0}\right\rangle\right)\right\rangle=-\left(h_{A}+h_{R}\right) \sigma_{00} \\
\left\langle A_{0}(x)\left(X_{1}-\left\langle X_{1}\right\rangle\right)\right\rangle=-\left(h_{A}+h_{R}\right) \sigma_{01} \\
\left\langle A_{0}(x)\left(X_{2}-\left\langle X_{2}\right\rangle\right)\right\rangle=-\left(h_{A}+h_{R}\right) \sigma_{02}, \\
\left\langle A_{1}(x)\left(X_{0}-\left\langle X_{0}\right\rangle\right)\right\rangle=-\left(k_{\mathrm{on}}+k_{\mathrm{off}}\right) \sigma_{01}, \\
\left\langle A_{1}(x)\left(X_{1}-\left\langle X_{1}\right\rangle\right)\right\rangle=-\frac{h_{A}\left(k_{\mathrm{on}}+k_{\mathrm{off}}\right)}{h_{R}+h_{A}} \sigma_{11}, \\
\left\langle A_{1}(x)\left(X_{2}-\left\langle X_{2}\right\rangle\right)\right\rangle=-\frac{\left(h_{A}+\gamma_{m}\right)\left(k_{\mathrm{on}}+k_{\mathrm{off}}\right)}{h_{R}+h_{A}+\gamma_{m}} \sigma_{12}, \\
\left\langle A_{2}(x)\left(X_{0}-\left\langle X_{0}\right\rangle\right)\right\rangle=\beta_{m} \sigma_{10}+\beta_{m}\left\langle X_{1}\right\rangle\left\langle X_{0}\right\rangle\left(1-\left\langle X_{0}\right\rangle\right)-\gamma_{m} \sigma_{20}, \\
\left\langle A_{2}(x)\left(X_{1}-\left\langle X_{1}\right\rangle\right)\right\rangle=\beta_{m}\left\langle X_{1} \sigma_{11}-\gamma_{m} \sigma_{21},\right. \\
\left\langle A_{2}(x)\left(X_{2}-\left\langle X_{2}\right\rangle\right)\right\rangle=\beta_{m} \frac{\left(h_{A}+\gamma_{m}\right)}{h_{R}+h_{A}+\gamma_{m}} \sigma_{12}+\beta_{m}\left\langle X_{1}\right\rangle\left(\left\langle X_{2}\right\rangle_{1}-\left\langle X_{2}\right\rangle\left\langle X_{0}\right\rangle\right) \\
\quad-\gamma_{m} \sigma_{22} .
\end{aligned}
$$

Therefore, at the stationary state, we obtain

$$
\left\langle A_{i}(X)\left(X_{k}-\left\langle X_{k}\right\rangle\right)\right\rangle+\left\langle A_{k}(X)\left(X_{i}-\left\langle X_{k}\right\rangle\right)\right\rangle+\left\langle B_{i k}(X)\right\rangle=0,
$$

which gives the following equations:

$$
\begin{aligned}
& 0=-2\left(h_{R}+h_{A}\right) \sigma_{00}+2 h_{R}\left\langle X_{0}\right\rangle \\
& 0=-\left(h_{R}+h_{A}\right) \sigma_{01}-\left(k_{\text {on }}+k_{\text {off }}\right) \sigma_{01} \\
& 0=-\left(h_{A}+h_{R}\right) \sigma_{02}-\gamma_{m} \sigma_{02}+\beta_{m}\left\langle X_{0}\right\rangle\left\langle X_{1}\right\rangle\left(1-\left\langle X_{1}\right\rangle\right) \\
& 0=-2 \frac{h_{A}\left(k_{\text {on }}+k_{\text {off }}\right)}{h_{R}+h_{A}} \sigma_{11}+2 k_{\text {off }}\left\langle X_{1}\right\rangle\left\langle X_{0}\right\rangle \\
& 0=-\frac{\left(h_{A}+\gamma_{m}\right)\left(k_{\text {on }}+k_{\text {off }}\right)}{h_{R}+h_{A}+\gamma_{m}} \sigma_{12}-\gamma_{m} \sigma_{12}+\beta_{m}\left\langle X_{0}\right\rangle \sigma_{11} \\
& 0=2\left(\beta_{m} \frac{\left(h_{A}+\gamma_{m}\right)}{h_{R}+h_{A}+\gamma_{m}} \sigma_{12}+\beta_{m}\left\langle X_{1}\right\rangle\left(\left\langle X_{2}\right\rangle_{1}-\left\langle X_{2}\right\rangle\left\langle X_{0}\right\rangle\right)-\gamma_{m} \sigma_{22}\right)+2 \gamma_{m}\left\langle X_{2}\right\rangle .
\end{aligned}
$$

Solving the above equations, we obtain $\sigma_{i j}$ :

$$
\begin{aligned}
\sigma_{00} & =\frac{h_{R}}{h_{R}+h_{A}}\left\langle X_{0}\right\rangle=\left\langle X_{0}\right\rangle\left(1-\left\langle X_{0}\right\rangle\right), \\
\sigma_{01} & =0
\end{aligned}
$$




$$
\begin{aligned}
& \sigma_{02}=\frac{\beta_{m}}{h_{R}+h_{A}+\gamma_{m}}\left\langle X_{2}\right\rangle \sigma_{00} \\
& \sigma_{11}=\frac{k_{\text {off }}}{k_{\text {on }}+k_{\text {off }}}\left\langle X_{1}\right\rangle \text {, } \\
& \sigma_{12}=\frac{\beta_{m}}{\frac{\left(h_{A}+\gamma_{m}\right)\left(k_{\text {on }}+k_{\text {off }}\right)}{h_{R}+h_{A}+\gamma_{m}}+\gamma_{m}}\left\langle X_{0}\right\rangle \sigma_{11}, \\
& \sigma_{22}=\left\langle X_{2}\right\rangle+\frac{\beta_{m}}{\gamma_{m}}\left(\frac{\left(h_{A}+\gamma_{m}\right)}{h_{R}+h_{A}+\gamma_{m}} \sigma_{12}+\left\langle X_{1}\right\rangle\left(\frac{\left(h_{A}+\gamma_{m}\right)}{h_{R}+h_{A}+\gamma_{m}}-\frac{h_{A}}{h_{R}+h_{A}}\right)\left\langle X_{2}\right\rangle\right) \\
& =\left\langle X_{2}\right\rangle+\frac{\beta_{m}}{\gamma_{m}}\left(\frac{\left(h_{A}+\gamma_{m}\right)}{h_{R}+h_{A}+\gamma_{m}} \frac{\beta_{m}}{\frac{\left(h_{A}+\gamma_{m}\right)\left(k_{\mathrm{on}}+k_{\mathrm{off}}\right)}{h_{R}+h_{A}+\gamma_{m}}+\gamma_{m}} \times\left\langle X_{0}\right\rangle \frac{k_{\mathrm{off}}}{k_{\mathrm{on}}+k_{\mathrm{off}}}\left\langle X_{1}\right\rangle\right. \\
& \left.+\left\langle X_{1}\right\rangle\left(\frac{\left(h_{A}+\gamma_{m}\right)}{h_{R}+h_{A}+\gamma_{m}}-\frac{h_{A}}{h_{R}+h_{A}}\right)\left\langle X_{2}\right\rangle\right) \text {. }
\end{aligned}
$$

Hence, let

$$
\theta=\frac{h_{R}}{h_{R}+h_{A}}, \quad k=\frac{k_{\mathrm{off}}}{k_{\mathrm{on}}+k_{\mathrm{off}}},
$$

and obtain

$$
\tau_{0}=\left(h_{R}+h_{A}\right)^{-1}, \quad \tau_{1}=\left(k_{\mathrm{on}}+k_{\mathrm{off}}\right)^{-1}, \quad \tau_{2}=\gamma_{N}^{-1},
$$

so we have

$$
\begin{aligned}
\eta_{0}^{2} & =\frac{\sigma_{00}}{\left\langle X_{0}\right\rangle^{2}}=\frac{h_{R}}{h_{R}+h_{A}} \frac{1}{\left\langle X_{0}\right\rangle}=\frac{\theta}{\left\langle X_{0}\right\rangle}, \\
\eta_{01} & =\frac{\sigma_{01}}{\left\langle X_{0}\right\rangle\left\langle X_{1}\right\rangle}=0, \\
\eta_{02} & =\frac{\sigma_{02}}{\left\langle X_{0}\right\rangle\left\langle X_{2}\right\rangle}=\frac{\tau_{0}}{\tau_{0}+\tau_{2}} \eta_{0}^{2}, \\
\eta_{1}^{2} & =\frac{\sigma_{11}}{\left\langle X_{1}\right\rangle^{2}}=\frac{k_{\text {off }}}{k_{\text {on }}+k_{\text {off }}} \frac{1}{\left\langle X_{1}\right\rangle}=\frac{k}{\left\langle X_{1}\right\rangle}, \\
\eta_{12} & =\frac{\sigma_{12}}{\left\langle X_{1}\right\rangle\left\langle X_{3}\right\rangle}=\frac{\tau_{2}}{\left(1-\frac{\theta}{1+\tau_{0} / \tau_{2}}\right) \tau_{3}+\tau_{2}} \eta_{1}^{2}, \\
\eta_{2}^{2} & =\frac{\sigma_{22}}{\left\langle X_{2}\right\rangle^{2}}=\frac{1}{\left\langle X_{2}\right\rangle}+\frac{1-\frac{\theta}{1+\tau_{0} / \tau_{2}}}{1-\theta} \eta_{12}+\left(1-\frac{1}{1+\tau_{0} / \tau_{2}}\right) \eta_{0}^{2} .
\end{aligned}
$$

The CV of mRNA products is given by $\eta_{2}^{2}$, which is an increasing function of $\theta$.

\section{References}

1. B. E. Bernstein et al., Cell 125, 315 (2006).

2. L. Bintu et al., Science 351, 720 (2016).

3. A. Cavazza et al., Stem Cell Rep. 6, 618 (2016). 
4. K. Cui et al., Stem Cell 4, 80 (2009).

5. I. B. Dodd et al., Cell 129, 813 (2007).

6. D. J. Gaffney et al., PLoS Genet. 8, e1003036 (2012).

7. P. Hajkova et al., Nature 452, 877 (2008).

8. K. Hayashi et al., Stem Cell 3, 391 (2008).

9. M. Kærn et al., Natl. Rev. Genet. 6, 451 (2005).

10. R. J. Klose and Y. Zhang, Natl. Rev. Mol. Cell Biol. 8, 307 (2007).

11. T. Kouzarides, Cell 128, 693 (2007).

12. W. L. Ku et al., PLoS ONE 8, e77944 (2013).

13. M. Lachner, R. J. O'Sullivan and T. Jenuwein, J. Cell Sci. 116, 2117 (2003).

14. D. Lara-Astiaso et al., Science 345, 943 (2014).

15. B. Lehnertz et al., Curr. Biol. 13, 1192 (2003).

16. J. Lei, J. Theor. Biol. 256, 485 (2009).

17. I. C. Macaulay, Cell Rep. 14, 1 (2016).

18. H. D. Morgan et al., Natl. Genet. 23, 314 (1999).

19. S. Mukhopadhyay and A. M. Sengupta, PLoS Comput. Biol. 9, e1003121 (2013).

20. A. V. Probst, E. Dunleavy and G. Almouzni, Natl. Rev. Mol. Cell Biol. 10, 192 (2009).

21. A. Richard et al., PLoS Biol. 14, e1002585 (2016).

22. A. J. Ruthenburg, C. D. Allis and J. Wysocka, Mol. Cell 25, 15 (2006).

23. X. Shen and S. H. Orkin, Stem Cell 4, 1 (2008).

24. K. Sneppen and I. B. Dodd, PLoS Comput. Biol. 8, e1002643 (2011).

25. K. Sneppen, M. A. Micheelsen and I. B. Dodd, Mol. Syst. Biol. 4, 182 (2007).

26. Y. Song, H. Ren and J. Lei, Int. J. Mod. Phys. B 1, 1750243 (2017).

27. W. Wu et al., Genome Res. 21, 1659 (2011).

28. H. Zhang et al., Phys. Rev. Lett. 112, 068101 (2014).

29. H. Zheng et al., Mol. Cell 63, 1066 (2016). 